\title{
The clinical and molecular genetic study of 20 Silver Russell Syndrome cases
}

\author{
Chunxiu Gong*, Ming-Qiang Zhu, Di Wu, Bingyan Cao \\ From 7th APPES Biennial Scientific Meeting \\ Nusa Dua, Bali. 14-17 November 2012
}

\section{Objective}

To analyze the genetic pathogenesis and improve the accuracy of the diagnosis of the disease, this study reported the clinical features of 20 patients with Silver Russell Syndrome (SRS) in the Beijing Children's Hospital and detected the chromosome 11p15 imprinting defects in 16 patients of them.

\section{Methods}

20 SRS cases diagnosed in Beijing Children's Hospital from 2006 to 2011 were studied retrospectively for clinical manifestations, physical signs, laboratory examinations and respond of $\mathrm{GH}$ treatment. We compared with 3 different diagnostic criteria and used the methylationspecific multiplex ligation dependent probe amplification (MS-MLPA) method to detect the chromosome 11p15 imprinting defects in 16 patients of them, meanwhile take 10 normal control.

\section{Results}

We collected 20 SRS patients over a period from 2006 to 2011 with 3 criteria. The concordance is $90 \%$. Include fifteen males and five females, age range $0.08 \sim 12.17 \mathrm{yr}$. The most chief complaint is short, $85 \%$. Then, it is asymmetry (5\%), and external genital abnormalities (10\%). The clinical characteristics with the frequencies accounted for over $80 \%$ included small for gestation age(SGA), postnatal growth retardation, craniofacial dysmorphism, asymmetry and super thin of whole body, extremely limbs, fifth finger clinodactyly, BMI<-2SDS and height was obviously lagging hypomethylation and ICR2 hypermethylation may be the result of the maternal chromosome 11p15 uniparental disomy. Another case had duplication of the maternal chromosome 11p15 fragment. Two cases had good effects of GH treatment, one with chromosome 11p15 ICR1 hypomethylation and the other one was normal.

\section{Conclusion}

The top 3 clinical features in SRS are (1) growth retardation include SGA and/or postnatal. (2) Malformation include craniofacial dysmorphism, asymmetry of face and/ or limbs, fifth finger clinodactyly. (3) Super severe low BMI and height was obviously lagging behind bone age. No laboratory and imagination specificity. Chromosome 11 p15 imprinting defect is the major genetic disturbance in SRS, about $50 \%$, and ICR1 hypomethylation is the predominant molecular alteration. The MS-MLPA is a technique for detecting all chromosome 11p15 imprinting defects of SRS. It is useful for the study of the genetic aspects of SRS. The relation between the respond of GH treatment and genetic changes is uncertainty.

Published: 3 October 2013

doi:10.1186/1687-9856-2013-S1-P45

Cite this article as: Gong et al:: The clinical and molecular genetic study of 20 Silver Russell Syndrome cases. International Journal of Pediatric Endocrinology 2013 2013(Suppl 1):P45. behind the bone age. Six of them had used the growth hormone for 3-months to 12 Months. The growth velocity is from $4 \mathrm{~cm}$ to $10 \mathrm{~cm} /$ year. In the 16 patients, 6 patients were found hypomethylation in chromosome 11p15 ICR1, the other one with chromosome $11 \mathrm{p} 15$ ICR1

Beijing Children's Hospital, The Capital University, Beijing, China 